\title{
Erratum: Metallic phases from disordered (2+1)-dimensional quantum electrodynamics [Phys. Rev. B 95, 235145 (2017)]
}

\author{
Pallab Goswami, Hart Goldman, and S. Raghu
}

(Received 25 January 2019; published 7 February 2019)

DOI: 10.1103/PhysRevB.99.079903

We correct an error in our paper. We show that, in the large- $N$ limit, the chemical potential disorder in (2+1)-dimensional quantum electrodynamics $\left(\mathrm{QED}_{3}\right)$ is screened even more strongly than we had originally written due to the presence of additional bubble diagrams. Consequently, the scaling dimension of the disorder is corrected so that it is more irrelevant. This correction is consistent with our results using the $\epsilon$ expansion in the appendix of that paper in the limit $N \rightarrow \infty$. In the original paper, we presented a perturbative study of the effects of quenched disorder on a theory of Dirac fermions strongly coupled to an emergent $U(1)$ gauge field or quantum electrodynamics in $(2+1)$ space-time dimensions $\left(\mathrm{QED}_{3}\right)$. We demonstrated that the strong interactions in these theories can stabilize metallic behavior and even lead to new metallic phases characterized by finite disorder and interaction strengths.

As a simple example of how a metallic phase can be stabilized by gauge fluctuations, we considered $\mathrm{QED}_{3}$ with a large number $j=1, \ldots, N$ of four-component Dirac fermion flavors $\Psi_{j}$ and a random chemical potential,

$$
\begin{gathered}
S=S_{0}+S_{\mathrm{dis}}, \\
S_{0}=-\int d^{2} \mathbf{x} d \tau\left[\bar{\Psi}_{j} \gamma^{\mu}\left(\partial_{\mu}-i \sqrt{\frac{\alpha}{N}} a_{\mu}\right) \Psi_{j}-\frac{1}{4} f_{\mu \nu} f^{\mu \nu}\right], \\
S_{\mathrm{dis}}=\int d^{2} \mathbf{x} d \tau V(\mathbf{x}) \bar{\Psi}_{j} \gamma^{0} \Psi_{j}(\mathbf{x}, \tau)=\int d^{2} \mathbf{x} d \tau V(\mathbf{x}) \Psi_{j}^{\dagger} \Psi_{j}(\mathbf{x}, \tau),
\end{gathered}
$$

where $a_{\mu}$ is a fluctuating $U(1)$ gauge field, $f_{\mu \nu}=\partial_{\mu} a_{\nu}-\partial_{\nu} a_{\mu}$, and we work in Euclidean space-time. $V(\mathbf{x})$ is a random scalar potential with correlations,

$$
\overline{V(\mathbf{x}) V\left(\mathbf{x}^{\prime}\right)}=\frac{\Delta}{\left|\mathbf{x}-\mathbf{x}^{\prime}\right| \chi}, \quad \overline{V(\mathbf{x})}=0 .
$$

where Gaussian white-noise disorder corresponds to $\chi \rightarrow 2$ (the number of spatial dimensions). Since $V(\mathbf{x})$ is a scalar potential, it should have mass dimension $[V]=1$. Thus, the dimension of the disorder strength $\Delta$ is

$$
[\Delta]=2-\chi,
$$

meaning that Gaussian white-noise disorder is marginal at the tree level in two dimensions.

In the large- $N$ limit, clean $\mathrm{QED}_{3}$ flows to a stable metallic infrared (IR) fixed point due to screening of the gauge-field propagator by fermion bubbles (see Appendix A of our paper for a review). In the presence of potential disorder, upon invoking the replica trick, we showed that this same screening effect corrects the disorder correlations and can be thought of as shifting $\chi \mapsto \chi_{\text {int }}=\chi-2 \eta$. In the original paper, we found, upon taking the $N \rightarrow \infty$ limit, that $\eta=-1 / 2$ and $[\Delta]=-1$ for chemical potential disorder, rendering it strongly irrelevant and stabilizing the metallic fixed point. However, in that work, there was an error in how the diagrams in question were summed. In fact, the correct results are $\eta=-1$ and $[\Delta]=-2$. This is consistent with the result obtained using the $\epsilon$ expansion in Appendix D 4 of our paper.

To see that this is the case, we perform disorder averaging using the replica trick, introducing $a=1, \ldots, n_{r}$ replicas of $\Psi$ and $a_{\mu}$, eventually taking the limit $n_{r} \rightarrow 0$. This leads to an effective disorder interaction which is nonlocal in imaginary time,

$$
S_{\mathrm{dis}}=-\frac{\Delta}{2} \int d^{2} \mathbf{x} d \tau d \tau^{\prime} \Psi_{a, i}^{\dagger} \Psi_{a, i}(\mathbf{x}, \tau) \Psi_{b, j}^{\dagger} \Psi_{b, j}\left(\mathbf{x}, \tau^{\prime}\right)
$$

At leading order in the large- $N$ expansion, this interaction is corrected by diagrams of the form given in Fig. 1 . However, these diagrams are not simply a geometric series as was assumed in the original paper since one has to account for all of the ways to arrange the internal photon and disorder propagators. As a result, this sum over diagrams is actually the square of a geometric 

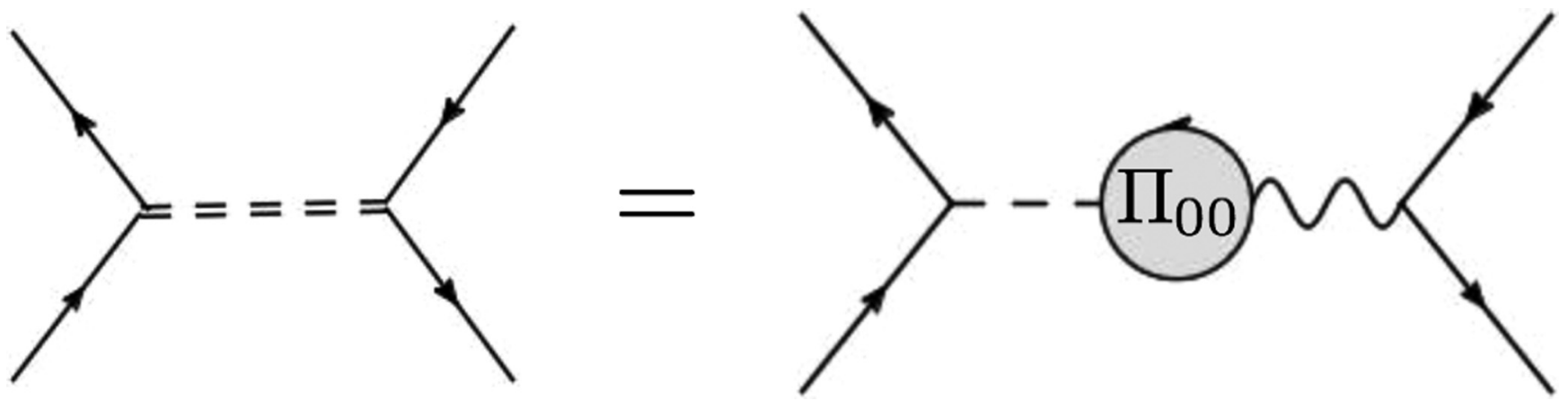

FIG. 1. Screening of the disorder at leading order in the large- $N$ expansion. Here, the solid lines are fermion propagators, the dashed lines are disorder propagators, and the wavy lines are gauge-field propagators.

series. To see this, consider the first few terms in the series,

$$
\begin{aligned}
\Gamma_{\text {dis }}=|\ldots|+|+| \\
=\Delta\left\{1-2 \Pi_{00}(\mathbf{p}, 0) /|\mathbf{p}|^{2}+3\left[\Pi_{00}(\mathbf{p}, 0)\right]^{2} /|\mathbf{p}|^{4}+\cdots\right\} \\
=\Delta\left(\frac{1}{1+\Pi_{00}(\mathbf{p}, 0) /|\mathbf{p}|^{2}}\right)^{2} \sim \frac{\Delta}{\alpha^{2}}|\mathbf{p}|^{2},
\end{aligned}
$$

where $\Pi_{\mu v}(\mathbf{p}, \omega)=\frac{\alpha}{8} p\left(\delta_{\mu \nu}-p_{\mu} p_{v} / p^{2}\right)$ is the polarization tensor (the value of the bubble integral). In the last line, we have taken the IR limit $p \ll \alpha$. Fourier transforming Eq. (9), the effective disorder correlations at the IR fixed point are

$$
\overline{V(\mathbf{x}) V\left(\mathbf{x}^{\prime}\right)} \sim \frac{\Delta}{\left|\mathbf{x}-\mathbf{x}^{\prime}\right|^{4}} .
$$

In other words, $\chi_{\text {int }}=4$, so $\eta=-1$ and $[\Delta]=-2$. This is in contrast to what is written, e.g., in Eq. (10) or our paper. Note that this does not change any of our essential conclusions (and, again, these results are consistent with the $\epsilon$ expansion) but rather speaks to the power of gauge fluctuations in suppressing disorder effects! 